\title{
Completing visual contours: The relationship between relatability and minimizing inflections
}

\author{
MANISH SINGH and DONALD D. HOFFMAN \\ University of Califormia, Irvine, Califormia
}

\begin{abstract}
Visual completion is a ubiquitous phenomenon: Human vision often constructs contours and surfaces in regions that have no sharp gradients in any image property. When does human vision interpolate a contour between a given pair of luminance-defined edges? Two different answers have been proposed: relatability and minimizing inflections. We state and prove a proposition that links these two proposals by showing that, under appropriate conditions, relatability is mathematically equivalent to the existence of a smooth curve with no inflection points that interpolates between the two edges. The proposition thus provides a set of necessary and sufficient conditions for two edges to be relatable. On the basis of these conditions, we suggest a way to extend the definition of relatability (1) to include the role of genericity, and (2) to extend the current all-or-none character of relatability to a graded measure that can track the gradedness in psychophysical data.
\end{abstract}

Visual completion is a process of human vision that can construct contours and surfaces in regions that have no sharp gradients in any image property. Researchers distinguish two kinds of visual completion (Michotte, Thines, \& Crabbe, 1964/1991). In modal completion, the perception of a completed surface is accompanied by a perceived brightness gradient along its completed contour. For example, in Kanizsa's (1979) illusory triangle (Figure 1b), the region inside the triangle is seen as being whiter than the surrounding white. In amodal completion, by contrast, there is no perceived brightness gradient, even though one is aware of the shape of the completed surface. For example, in Figure la, one is aware of a unified elliptical shape passing behind the rectangle-even though one sees no brightness edges in the region where the ellipse is occluded by the rectangle.

Human vision does not, however, always complete contours to form unified objects. In Figures $1 \mathrm{c}$ and $1 \mathrm{~d}$, for example, the displays of Figures $1 \mathrm{a}$ and $1 \mathrm{~b}$ have been respectively modified so that one no longer has the perception of unified, completed shapes. What spatial factors determine when human vision will, or will not, complete a visual contour?

This question has been addressed in the literature in two different ways. One proposal, owing to Kellman and Shipley (1991), is that the process of interpolation between two luminance-defined edges proceeds when the two edges are relatable to one another. The precise defi-

This work was supported in part by National Science Foundation Grant SBR-9014278. For discussions and suggestions, we thank Bruce Bennett, Myron Braunstein, Mike D'Zmura, and Chetan Prakash. We also thank Jan Drösler, Shinsuke Shimojo, and two anonymous reviewers for helpful comments on an earlier version of the paper. Address correspondence to M. Singh, Department of Brain and Cognitive Sciences, NE20-451, Massachusetts Institute of Technology, Cambridge, MA 02139 (e-mail: manish@psyche.mit.edu). nition of relatability is as follows (see Kellman \& Shipley, 1991, pp. 174-175):

DEFINITION 1. Let $E_{1}$ and $E_{2}$ be two surface edges, with $s_{1}$ and $s_{2}$ as their respective linear extensions (see Figure 2). Then the two edges are said to be relatable if (1.1) the two linear extensions $s_{1}$ and $s_{2}$ intersect; and (1.2) their outer angle of intersection (angle $\phi$ in Figure 2) is acute. 1

For example, in Figures $1 \mathrm{a}$ and $\mathrm{lb}$, the various edge pairs are relatable, whereas in Figures $1 \mathrm{c}$ and $1 \mathrm{~d}$, they are not. In a number of experimental studies, Shipley and Kellman (e.g., Shipley \& Kellman, 1992a, 1992b) have shown the psychological relevance of the notion of relatability. For example, they found that the strength of a perceived interpolated contour decreases steadily as a given edge pair deviates from perfect relatability (as in the displays in Figures $1 \mathrm{c}$ and 1d).

A different proposal, owing to Takeichi, Nakazawa, Murakami, and Shimojo (1995), involves the role of inflection points (i.e., points where a curve reverses its sign of curvature). According to this proposal, (1) human vision completes visual contours in a way that minimizes the number of inflection points on the interpolated contour, and (2) the more inflection points an interpolated contour needs to have (while maintaining continuity of direction), the lower its perceptual strength-and the less likely it is that visual completion will take place. ${ }^{2}$ Takeichi et al. have presented evidence that subjects indeed complete visual contours in a way that is consistent with these requirements. For the displays in Figures $1 \mathrm{c}$ and $1 \mathrm{~d}$, for example, any smooth curve interpolating between the various edge pairs must have at least one inflection point - and this might explain the absence, or weakness, of perceived interpolated contours in these figures. Takeichi et al. also note that modal completion (e.g., in illusory figures, such as Kanizsa's triangle) takes place only when it is possible to have a smooth interpolating curve that 


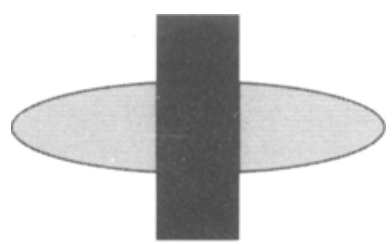

(a)

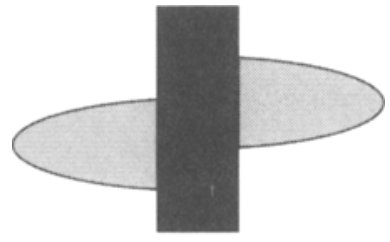

(c)

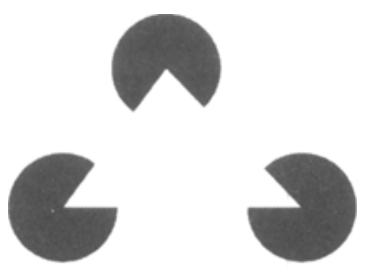

(b)

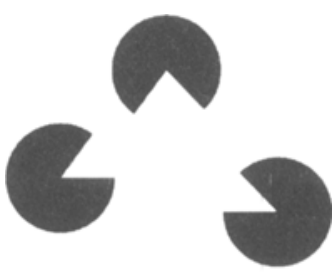

(d)
Figure 1. Two examples of visual completion: (a) amodal completion of an ellipse behind the rectangle, and (b) modal completion of a triangle in front of the disks. In (c) and (d) the perception of completed figures is greatly reduced.

has no points of inflection-hence modal completion may have a higher threshold than amodal completion. In addition to the requirement of minimizing inflection points, Takeichi et al. also require that the end points of the interpolated contour (i.e., the points where the interpolated contour meets the luminance-defined edges) not be points of inflection on the completed curve, and that the interpolated contour continue the curvature of the edges.

The theory of relatability and the theory of inflections, therefore, both provide a possible explanation for the same phenomenon-namely, the presence or absence, as well as the strength, of perceived interpolated contours. Furthermore, the two proposals seem related. For example, in Figures $1 \mathrm{c}$ and $1 \mathrm{~d}$, making the various edge pairs nonrelatable also introduces inflection points on any smooth curve interpolating between them. (Note, however, that the edge pairs in Figure 1 have the special property that the two edges in each pair are parallel.) In this paper, we address the question of what, in general, the precise relationship between these two proposals is.

In the next section, we state and prove a proposition which shows that, under appropriate conditions, relatability is in fact mathematically equivalent to the condition that there exists a smooth curve with no inflection points that interpolates between the two edges. This proposition therefore provides a set of necessary and sufficient conditions (and hence, an alternative definition) for relatability, in terms of properties that an interpolating curve must satisfy - and this includes the property of having no inflections. Following this, we consider some other points of difference between the theoretical framework of Kellman and Shipley (1991) and that of Takeichi et al.
(1995). We end with some constructive suggestions on possible ways to extend the notion of relatability.

\section{RELATABILITY AND INFLECTIONS: A MATHEMATICAL RESULT}

In Kellman and Shipley's definition of relatability, it is clear that an edge is essentially thought of as an ordered pair $(\mathbf{a}, \mathbf{t})$, where $\mathbf{a}$ is a point in $\Re^{2}$ (the point at which interpolation must begin), and a unit direction vector $t$ (giving the direction of the edge at point a), along which the interpolated contour must continue. So, for example, neither the length of the edge, nor its curvature, affects the definition of relatability (see Definition 1 ). ${ }^{3}$

For any such ordered pair $(\mathbf{a}, \mathbf{t})$, with $\mathbf{a}, \mathbf{t} \in \mathfrak{R}^{2}$ and $\|\mathbf{t}\|=1$, let $R(\mathbf{a}, \mathbf{t})$ denote the open ray originating at $\mathbf{a}$, and in the direction of $t$. That is,

$$
R(\mathbf{a}, \mathbf{t})=\left\{\mathbf{x} \in \mathfrak{R}^{2} \mid \mathbf{x}=\mathbf{a}+r \mathbf{t} ; r>0\right\} .
$$

Then the definition of relatability can be reformulated as follows.

DEFINITION 1a. Let $\mathbf{a}_{1}, \mathbf{a}_{2}, \mathbf{t}_{1}, \mathbf{t}_{2} \in \Re^{2}$, with $\left\|\mathbf{t}_{1}\right\|=\left\|\mathbf{t}_{2}\right\|=1$. The two edges $\left(\mathbf{a}_{1}, \mathbf{t}_{1}\right)$ and $\left(\mathbf{a}_{2}, \mathbf{t}_{2}\right)$ are relatable (see Figure 3) if

$$
\text { (1a.1) } R\left(\mathbf{a}_{1}, \mathbf{t}_{1}\right) \cap R\left(\mathbf{a}_{2}, \mathbf{t}_{2}\right) \neq \varnothing \text {, }
$$

and

$$
\text { (1a.2) }|\phi|<\frac{\pi}{2}
$$

where $\phi$ is the directed angle from $\mathbf{t}_{1}$ to $-\mathbf{t}_{2}$.

With this simple reformulation, we can now state the proposition. Recall that a $C^{1}$ curve is one that is first-order continuous everywhere; and a piecewise $C^{2}$ curve is one

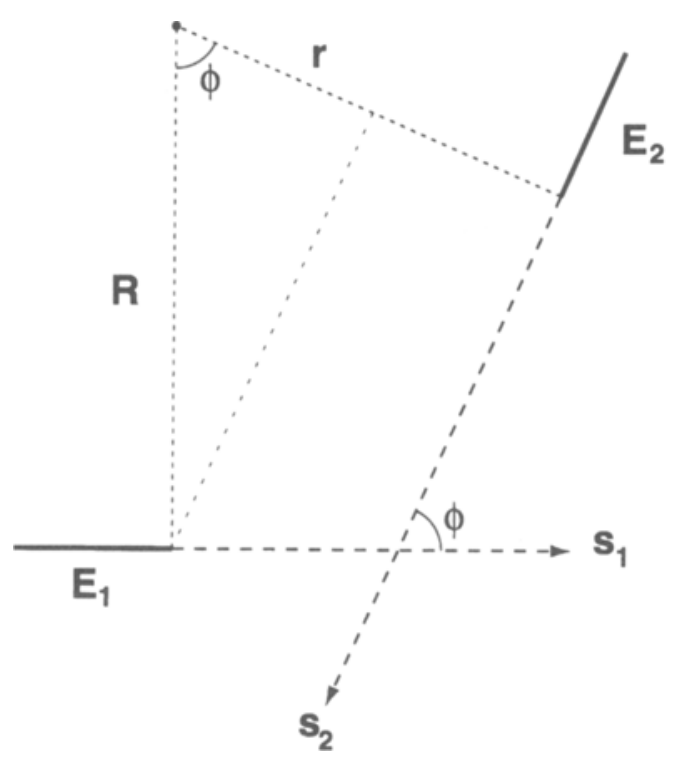

Figure 2. Kellman and Shipley's (1991) definition of relatability: The linear extensions of the two edges must meet, and their outer angle of intersection must be acute. 


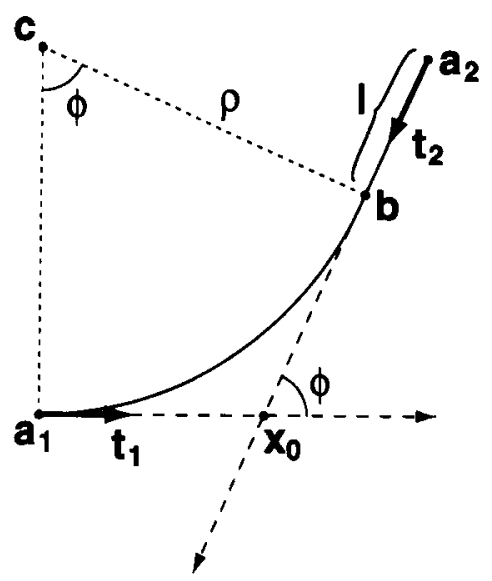

Figure 3. A $C^{1}$ and piecewise $C^{2}$ curve that interpolates between two relatable edges (Kellman \& Shipley, 1991, Appendix B). This curve satisfies the conditions of our proposition.

that is second-order continuous everywhere except, possibly, for finitely many points.

Proposition. Two edges $\left(\mathbf{a}_{1}, \mathbf{t}_{1}\right)$ and $\left(\mathbf{a}_{2}, \mathbf{t}_{2}\right)$ are relatable if and only if there exists a regular $C^{1}$, and piecewise $C^{2}$, plane curve $\alpha:[0, L] \rightarrow \Re^{2}$, with curvature $\kappa_{\alpha}$, that satisfies the following three properties: (1) $\alpha(0)=\mathbf{a}_{1}$; $\alpha(L)=\mathbf{a}_{2} ; \alpha^{\prime}(0)=\mathbf{t}_{1} ; \alpha^{\prime}(L)=-\mathbf{t}_{2} ;(2) \kappa_{\alpha}(s)$ does not reverse sign for $0<s<L$; and (3) $\int_{0}^{L}\left|\kappa_{\alpha}(s)\right| d s<\pi / 2$.

Condition 1 simply says that the interpolating curve must start at the point $\mathbf{a}_{1}$, with tangent $\mathbf{t}_{1}$, and terminate at the point $\mathbf{a}_{2}$, with tangent $-\mathbf{t}_{2}$. Condition 2 says that the curve should have no points of inflection. Condition 3 says that the interpolating curve must not turn through a total angle of more than $\pi / 2$. We will comment further on Condition 3 immediately following the proof of the proposition.

ProOf. (I) Let us first assume that the two edges $\left(\mathbf{a}_{1}, \mathbf{t}_{1}\right)$ and $\left(a_{2}, t_{2}\right)$ are relatable. We need to show the existence of a $C^{1}$, and piecewise $C^{2}$, plane curve $\alpha$ that interpolates between these two edges, and satisfies Properties 1-3 of the proposition.

If $\mathbf{t}_{1}$ and $\mathbf{t}_{2}$ are parallel, relatability ( 1$)$ forces $R\left(\mathbf{a}_{1}, \mathbf{t}_{1}\right)$ and $R\left(\mathbf{a}_{2}, \mathbf{t}_{2}\right)$ to be colinear, for otherwise $R\left(\mathbf{a}_{1}, \mathbf{t}_{1}\right) \cap$ $R\left(\mathbf{a}_{2}, \mathbf{t}_{2}\right)=\varnothing$; and (2) forces $\phi=0$, for otherwise Condition 2 of Definition la is violated. In this case, we can simply define $\alpha$ to be the straight-line segment joining $\mathbf{a}_{1}$ to $\mathbf{a}_{2}$, as follows,

$$
\alpha(s)=\mathbf{a}_{1}+s \mathbf{t}_{1}, s \in[0, L],
$$

where $L=\left\|\mathbf{a}_{1}-\mathbf{a}_{2}\right\|$. Then $\alpha$ trivially satisfies Properties $1-3$ of the proposition.

If $t_{1}$ and $t_{2}$ are not parallel, we use the construction of Skeath (Kellman \& Shipley, 1991, Appendix B), ${ }^{4}$ and show that this curve satisfies the three properties.

Assume without loss of generality that $\mathbf{a}_{1}=\mathbf{0}$, the origin of the coordinate system, and $\mathbf{t}_{1}=\mathbf{i}=(1,0)$, the unit vector along the positive $x$-direction. Then, $-t_{2}=(\cos \phi$, $\sin \phi$ ). Invoking Property 1 of relatability, let $R\left(\mathbf{a}_{1}, \mathbf{t}_{1}\right) \cap$ $R\left(\mathbf{a}_{2}, \mathbf{t}_{2}\right)=\left\{\mathbf{x}_{0}\right\}$. (Two nonparallel rays either are disjoint or intersect in a point.) Assume also that $\| \mathbf{a}_{1}-$ $\mathbf{x}_{0}\|\leq\| \mathbf{a}_{2}-\mathbf{x}_{0} \|$ and $\phi>0$. (The proofs in the other cases are similar.)

The curve $\alpha$ interpolating from $\mathbf{a}_{1}$ to $\mathbf{a}_{2}$ is now constructed by piecing together an arc of a circle and a straight-line segment (see Figure 3), as follows.

If $\left\|\mathbf{a}_{1}-\mathbf{x}_{0}\right\|<\left\|\mathbf{a}_{2}-\mathbf{x}_{0}\right\|$, there exists a point $\mathbf{b}$ on $R\left(\mathbf{a}_{2}, \mathbf{t}_{2}\right)$, lying between $\mathbf{a}_{2}$ and $\mathbf{x}_{0}$, such that $\left\|\mathbf{a}_{1}-\mathbf{x}_{0}\right\|=$ $\left\|\mathbf{b}-\mathbf{x}_{0}\right\|$. If $\left\|\mathbf{a}_{1}-\mathbf{x}_{0}\right\|=\left\|\mathbf{a}_{2}-\mathbf{x}_{0}\right\|$, take $\mathbf{b}=\mathbf{a}_{2}$. Let $\mathbf{t}_{3}=$ $\mathbf{j}=(0,1)$, the unit vector along the positive $y$-direction, and

$$
\mathbf{t}_{4}=\left[\cos \left(\phi+\frac{\pi}{2}\right), \sin \left(\phi+\frac{\pi}{2}\right)\right] .
$$

Then, the two rays $R\left(\mathbf{a}_{1}, \mathbf{t}_{3}\right)$ and $R\left(\mathbf{b}, \mathbf{t}_{4}\right)$ must intersect. Call their point of intersection $c$. Then, by our construction, we have

$$
\left\|\mathbf{a}_{1}-\mathbf{c}\right\|=\|\mathbf{b}-\mathbf{c}\|(=\rho),
$$

since the two triangles $\Delta \mathbf{c x _ { 0 }} \mathbf{a}_{1}$ and $\Delta \mathbf{c x _ { 0 }} b$ are congruent. Also, let $\left\|\mathbf{b}-\mathbf{a}_{2}\right\|=l$.

Now, define $\alpha:[0, L] \rightarrow \Re^{2}$ by:

$$
\alpha(s)= \begin{cases}\mathbf{c}+\rho\left[\cos \left(\frac{3 \pi}{2}+\frac{s}{\rho}\right), \sin \left(\frac{3 \pi}{2}+\frac{s}{\rho}\right)\right], & 0 \leq s \leq \rho \phi, \\ \mathbf{b}-(s-\rho \phi) \mathbf{t}_{2}, & \rho \phi \leq s \leq \rho \phi+l,\end{cases}
$$

where $L=\rho \phi+l$.

Then, we have

$$
\alpha^{\prime}(s)=\left\{\begin{array}{lr}
{\left[-\sin \left(\frac{3 \pi}{2}+\frac{s}{\rho}\right), \cos \left(\frac{3 \pi}{2}+\frac{s}{\rho}\right)\right],} & 0<s<\rho \phi, \\
-\mathbf{t}_{2}, & \rho \phi<s<L .
\end{array}\right.
$$

Therefore,

$$
\begin{aligned}
& \alpha(0)=\mathbf{c}+\rho\left(\cos \frac{3 \pi}{2}, \sin \frac{3 \pi}{2}\right)=\mathbf{a}_{1}, \\
& \alpha(L)=\mathbf{b}-(L-\rho \phi) \mathbf{t}_{2}=\mathbf{b}-l \mathbf{t}_{2}=\mathbf{a}_{2}, \\
& \alpha^{\prime}(0)=\left(-\sin \frac{3 \pi}{2}, \cos \frac{3 \pi}{2}\right)=(1,0)=\mathbf{t}_{1}, \\
& \alpha^{\prime}(L)=-\mathbf{t}_{2} .
\end{aligned}
$$

Clearly, $\alpha$ is unit speed $\left(\left\|\alpha^{\prime}(s)\right\|=1\right.$ for $\left.0<s<L\right)$, and it is $C^{2}$ on each of its two components (arc of circle, and straight-line segment). Also, letting $\alpha_{-}$denote the left-hand limit and $\alpha_{+}$denote the right-hand limit, then

$$
\begin{aligned}
\alpha_{-}(\rho \phi) & =\mathbf{c}+\rho\left[\cos \left(\frac{3 \pi}{2}+\phi\right), \sin \left(\frac{3 \pi}{2}+\phi\right)\right]=\mathbf{b}, \\
\alpha_{+}(\rho \phi) & =\mathbf{b}, \\
\alpha_{-}^{\prime}(\rho \phi) & =\left[-\sin \left(\frac{3 \pi}{2}+\phi\right), \cos \left(\frac{3 \pi}{2}+\phi\right)\right] \\
& =(\cos \phi, \sin \phi)=-\mathbf{t}_{2}, \\
\alpha_{+}^{\prime}(\rho \phi) & =-\mathbf{t}_{2} .
\end{aligned}
$$


Hence, $\alpha^{\prime}$ is continuous at $s=\rho \phi$, and therefore $\alpha$ is $C^{1}$, and piecewise $C^{2}$, on $[0, L](s=\rho \phi$ is the only point of $C^{2}$ discontinuity for $0<s<L$ ).

Since $\alpha$ is a unit speed curve, the plane curvature of $\alpha$ is defined by

$$
\kappa_{\alpha}(s)=\theta^{\prime}(s)
$$

where $\theta(s)$ is the slope angle of $\alpha$ at $s .^{5}$

We have,

$$
\theta(s)=\left\{\begin{array}{l}
s / \rho, 0 \leq s \leq \rho \phi \\
\phi, \quad \rho \phi \leq s \leq L
\end{array} .\right.
$$

Therefore,

$$
\kappa_{\alpha}(s)=\left\{\begin{array}{ll}
1 / \rho, & 0<s<\rho \phi \\
0, & \rho \phi<s<L
\end{array} .\right.
$$

Hence $\kappa_{\alpha}(s)$ does not reverse sign for $0<s<L$.

Also,

$$
\int_{0}^{L}\left|\kappa_{\alpha}(s)\right| d s=\int_{0}^{\rho \phi} \frac{1}{\rho} d s=\frac{1}{\rho}(\rho \phi)=\phi,
$$

and we know, by relatability, that

$$
\phi<\frac{\pi}{2} \text {. }
$$

This proves the first half of the proposition.

(II) Let us now assume that we are given a $C^{1}$, and piecewise $C^{2}$, curve $\alpha:[0, L] \rightarrow \Re^{2}$ which interpolates between two edges $\left(\mathbf{a}_{1}, \mathbf{t}_{1}\right)$ and $\left(\mathbf{a}_{2}, \mathbf{t}_{2}\right)$, and satisfies Properties $1-3$ of the proposition. We can assume that $\alpha$ is unit speed. (Otherwise, it is always possible to reparametrize $\alpha$ to make it unit speed.) We show that $\left(\mathbf{a}_{1}, \mathbf{t}_{1}\right)$ and $\left(\mathbf{a}_{2}, \mathbf{t}_{2}\right)$ must be relatable.

Assume, as before, that $\mathbf{a}_{1}=\mathbf{0}$, and $\mathbf{t}_{1}=\mathbf{i}=(1,0)$. Since $\alpha$ is $C^{1}$, it is possible to define a continuous function $\theta$ on $[0, L]$, such that $\theta(s)$ gives the slope angle of $\alpha$ at $s$ [with $\theta(0)=0$ ]. Since $\alpha$ is piecewise $C^{2}, \kappa_{\alpha}(s)=\theta^{\prime}(s)$ is defined for all but finitely many points in $[0, L]$. By Property 2 , since $\kappa_{\alpha}(s)$ does not reverse sign, let us assume, without loss of generality, that $\kappa_{\alpha}(s) \geq 0, \forall s \in$ $[0, L]$ [wherever $\kappa_{\alpha}(s)$ is defined]. Hence, $\theta$ is monotonically increasing on $[0, L]$.

Let $\theta(L)=\phi$. By Property 1 of the proposition, we must have $-t_{2}=\alpha^{\prime}(L)=(\cos \phi, \sin \phi)$, so that

$$
\phi=\cos ^{-1}\left(\mathbf{t}_{1} \cdot-\mathbf{t}_{2}\right) .
$$

Since $\theta(0)=0$, and $\theta$ is monotonically increasing on $[0, L]$, it follows that $\phi \geq 0$. If $\phi=0$, it must be the case, by monotonicity, that $\theta(s)=0, \forall s \in[0, L]$, so that $\alpha$ is a straightline segment. In this case, $\left(\mathbf{a}_{1}, \mathbf{t}_{1}\right)$ and $\left(\mathbf{a}_{2}, \mathbf{t}_{2}\right)$ are trivially relatable. Let us assume, therefore, that $\phi>0$.

By Property 3 of the proposition, we have

$$
\int_{0}^{L} \theta^{\prime}(s) d s<\frac{\pi}{2}
$$

since $\kappa_{\alpha}(s)=\theta^{\prime}(s) \geq 0$ for $0 \leq s \leq L$ [except, of course, for the finitely many points where $\theta^{\prime}(s)$ is not defined- but these do not affect the integral]. By Cauchy's fundamental theorem of calculus, we have

$$
\int_{0}^{L} \theta^{\prime}(s) d s=\theta(L)-\theta(0)=\phi .
$$

Therefore,

$$
0<\phi=\cos ^{-1}\left(\mathbf{t}_{1} \cdot-\mathbf{t}_{2}\right)<\frac{\pi}{2},
$$

and this proves the second condition in the definition of relatability.

Now, we show that the two open rays $R\left(\mathbf{a}_{1}, \mathbf{t}_{1}\right)$ and $R\left(\mathbf{a}_{2}, \mathbf{t}_{2}\right)$ must intersect. Let $\mathbf{a}_{2} /\left\|\mathbf{a}_{2}\right\|=(\cos \beta, \sin \beta)$. Hence $\beta$ is the slope of the chord joining $\mathbf{a}_{1}$ and $\mathbf{a}_{2}$ (recall that $\mathbf{a}_{1}=\mathbf{0}$ ). It is clear (see Figure 4 ) that $R\left(\mathbf{a}_{1}, \mathbf{t}_{1}\right)$ and $R\left(\mathbf{a}_{2}, \mathbf{t}_{2}\right)$ will intersect if and only if

$$
\beta<\phi<\pi \text {. }
$$

We have already shown that

$$
\phi<\frac{\pi}{2} \text {. }
$$

Hence we need only show that $\beta<\phi$.

Since $\alpha$ is $C^{1}$, we know by the mean value theorem that there exists an $s_{0}$ in the open interval $(0, L)$ such that $\alpha^{\prime}\left(s_{0}\right)$ is parallel to the chord joining $\mathbf{a}_{1}$ and $\mathbf{a}_{2}$. In other words,

$$
\exists s_{0} \in(0, L): \theta\left(s_{0}\right)=\beta .
$$

Since $\theta$ is monotonically increasing on $[0, L]$, we must have

$$
\beta=\theta\left(s_{0}\right) \leq \theta(L)=\phi .
$$

To start a reductio ad absurdum, assume that $\beta=\phi$. Then, we must have (again, by the monotonicity of $\theta$ ),

$$
\theta(s)=\beta, \forall s \in\left[s_{0}, L\right] .
$$

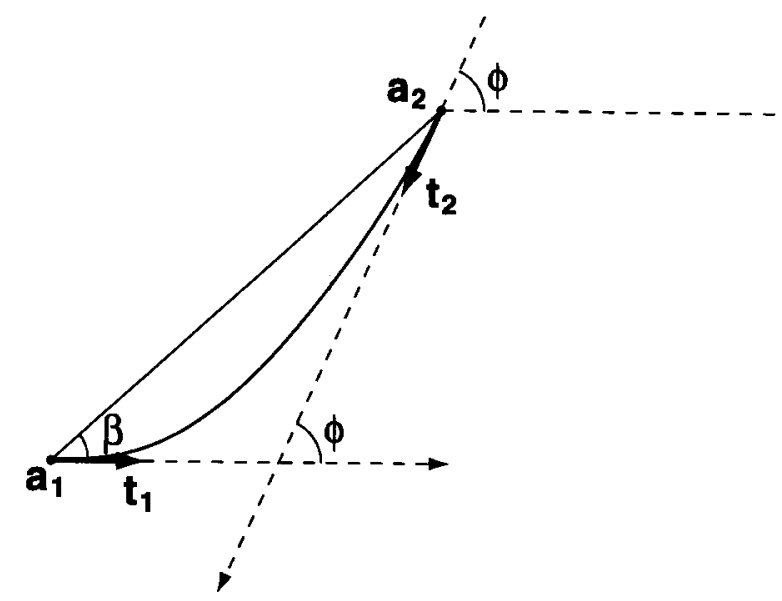

Figure 4. Two open rays interesect if and only if $\beta<\phi<\pi$. 
Define the set,

$$
T=\{t \in[0, L) \mid \theta(s)=\beta, \forall s \in[t, L]\} .
$$

Since $s_{0} \in T$, we know that $T \neq \varnothing$. Also, it is clear that $T$ is a closed set, and it is bounded below since $0 \notin T$ [recall that $\theta(0)=0]$. Therefore, $T$ must have a minimal element. Let

$$
t^{*}=\min T(>0) \text {. }
$$

Since $t^{*} \in T, \alpha\left(t^{*}\right)$ lies on the chord joining $\mathbf{a}_{1}$ and $\mathbf{a}_{2}$, and therefore $\beta$ is also the slope of the chord joining $\mathbf{a}_{1}$ and $\alpha\left(t^{*}\right)$. Again applying the mean value theorem, we conclude that

$$
\exists s_{0}^{\prime} \in\left(0, t^{*}\right): \theta\left(s_{0}^{\prime}\right)=\beta .
$$

Now we have $s_{0}^{\prime}<t^{*}$ with

$$
\theta\left(s_{0}^{\prime}\right)=\theta\left(t^{*}\right)=\beta \text {. }
$$

Since $\theta$ is monotonic, this forces

$$
\theta(s)=\beta, \forall s \in\left[s_{0}^{\prime}, t^{*}\right],
$$

and therefore,

$$
\theta(s)=\beta, \forall s \in\left[s_{0}^{\prime}, L\right] .
$$

But this means that $s_{0}^{\prime} \in T$, which is a contradiction, since $t^{*}=\min T$, and $s_{0}^{\prime}<t^{*}$. This contradiction proves that $\beta<\phi$, and hence, the two open rays $R\left(\mathbf{a}_{1}, \mathbf{t}_{1}\right)$ and $R\left(\mathbf{a}_{2}, \mathbf{t}_{2}\right)$ must intersect.

\section{Some Remarks}

1. Condition 3 of the proposition-namely,

$$
\int_{0}^{L}\left|\kappa_{\alpha}(s)\right| d s<\frac{\pi}{2}
$$

-is strictly stronger than Condition 2 in the definition of relatability (Definition la)-namely,

$$
|\phi|<\frac{\pi}{2}
$$

Whereas $|\phi|$ measures the magnitude of the angle between the two tangents $\alpha^{\prime}(0)=\mathbf{t}_{1}$ and $\alpha^{\prime}(L)=-\mathbf{t}_{2}, \int_{0}^{L}|\kappa(s)|$ $d s$ measures the total angle through which the curve $\alpha$ turns as it traverses its path from $s=0$ to $s=L$. Figure 5 shows three examples that clarify this difference. For the curve in Figure 5a,

$$
\phi=0\left(<\frac{\pi}{2}\right)
$$

but

$$
\int_{0}^{L}\left|\kappa_{\alpha}(s)\right| d s=\pi\left(>\frac{\pi}{2}\right) .
$$

For the curve in Figure 5b,

$$
\phi=\frac{\pi}{4}\left(<\frac{\pi}{2}\right)
$$

but

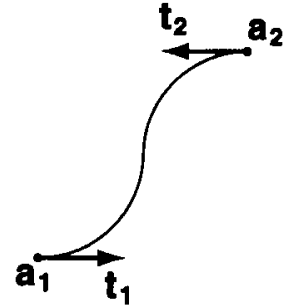

(a)

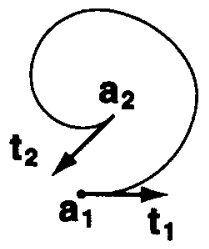

(c)

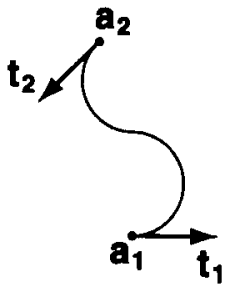

(b)

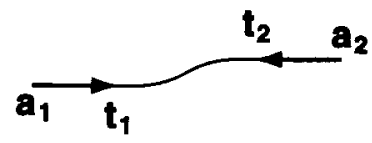

Figure 5. Examples that clarify the role of $\int_{0}^{L}\left|\kappa_{\alpha}(s)\right| d s$ in the proposition.

$$
\int_{0}^{L}\left|\kappa_{\alpha}(s)\right| d s=\frac{7 \pi}{4}\left(>\frac{\pi}{2}\right) .
$$

And for the curve in Figure 5c,

$$
\phi=\frac{\pi}{4}\left(<\frac{\pi}{2}\right)
$$

but

$$
\int_{0}^{L}\left|\kappa_{\alpha}(s)\right| d s=\frac{9 \pi}{4}\left(>\frac{\pi}{2}\right)
$$

2. The proposition is no longer true if Condition 3 ,

$$
\int_{0}^{L}\left|\kappa_{\alpha}(s)\right| d s<\frac{\pi}{2}
$$

is replaced with

$$
|\phi|<\frac{\pi}{2}
$$

A counterexample is provided by Figure $5 \mathrm{c}$; this satisfies

$$
|\phi|<\frac{\pi}{2},
$$

and the interpolating curve has no inflections, but the two edges are nevertheless not relatable.

3. The proposition is no longer true if Condition 2 (i.e., having no inflections) is removed. A counterexample is provided by Figure $5 \mathrm{~d}$, in which the interpolating curve satisfies 


$$
\int_{0}^{L}\left|\kappa_{\alpha}(s)\right| d s=\frac{\pi}{3}\left(<\frac{\pi}{2}\right),
$$

but the two edges are nevertheless not relatable.

4. Kellman and Shipley have also extended the definition of relatability to three dimensions, by adding the requirement that the two edges be coplanar. The proposition is easily extended to the 3-D case: One need only add a fourth condition requiring that the interpolating curve $\alpha$ be planar (i.e., have torsion 0 ).

\section{EXTENDING RELATABILITY}

One should be cautious in interpreting the proposition proved above. Although it provides a precise statement of the link between relatability and the role of inflection points, it should not be construed as claiming that the frameworks of Kellman and Shipley (1991) and Takeichi et al. (1995) are equivalent. One difference between the two frameworks, of course, is that Kellman and Shipley's definition of relatability requires that

$$
|\phi|<\frac{\pi}{2},
$$

whereas Takeichi et al.'s framework does not. Another difference, however, is that the framework of Takeichi et al. explicitly takes into account the role of genericity.

Genericity is a powerful constraint used by human vision. Like other visual constraints, it serves to reduce the countless number of interpretations that are always consistent with any given images. In its simplest form, this principle says to reject unstable interpretations of visual stimuli-those interpretations which, if perturbed slightly, would lead to a qualitative change in the image. It has been applied to provide theories of a number of visual capacities, such as the 3-D interpretation of line drawings (Binford, 1981), the perception of subjective contours (Albert \& Hoffman, 1995), the perception of object parts (Biederman, 1987; Hoffman, 1998; Hoffman \& Richards, 1984; Hoffman \& Singh, 1997; Singh \& Hoffman, 1997; Singh, Seyranian, \& Hoffman, 1999), the perception of shape from shading (Freeman, 1994), the phenomenon of color constancy (Brainard \& Freeman, 1997), and the perception of transparency (Singh \& Hoffman, 1998).

As pointed out earlier, the definition of relatability (Definition 1, or 1a) does not take into account the curvature of an edge - only its direction, and the location of the point where interpolation must begin. This allows for the possibility that the point where the interpolated curve meets the luminance-defined edge may be (1) a point of inflection (see Figure 6a), or (2) a point of discontinuity of curvature (i.e., a second-order discontinuity) of the completed contour (see Figure $6 \mathrm{c}$ ). ${ }^{6}$ Both of these cases are nongeneric (Takeichi et al., 1995). For example, assume that the end points of the interpolated curve are points of inflection on the completed contour (as in Figure 6a). In this case, a slight change in viewing position would perturb the occluding surface and the occluded surface slightly

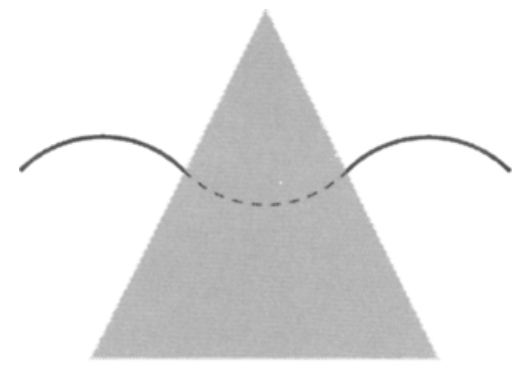

(a)

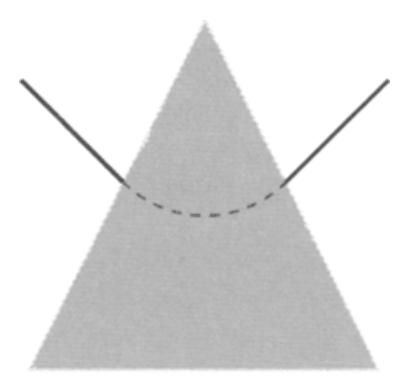

(c)

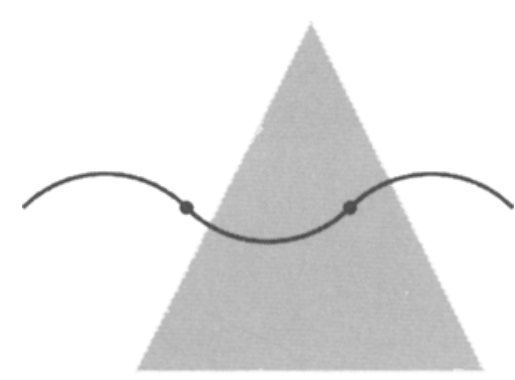

(b)

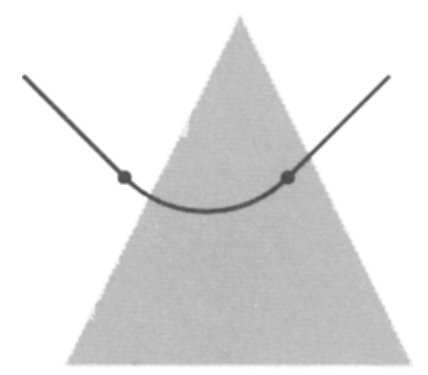

(d)

Figure 6. It is nongeneric for the end points of an interpolated curve to be points of inflection, or $C^{2}$ discontinuity, on the completed contour. 
with respect to each other-so that the points where occlusion begins would no longer be points of inflection (see Figure 6b). Another way to think of this is as follows: If we have two surfaces, one occluding the other, and the contour of the occluded surface has points of inflection on it, then only for a few special viewing positions will such an inflection point be a point where occlusion begins. Similarly, it is nongeneric for the end points of the interpolated curve to be points of $C^{2}$ discontinuity on the completed contour (see Figure 6d). However, it is less clear to what extent human vision is sensitive to $C^{2}$ discontinuities.

Future work on relatability needs to address these issues. One possibility might be to take the conditions of the proposition proved above as an alternate definition of relatability, and then to extend relatability by adding further conditions that an interpolating curve must satisfy. Some possible examples of such conditions are as follows: The end points of the interpolating curve should not be points of inflection, and the interpolating curve should be $C^{2}$ continuous everywhere, including at the endpoints. In order to do this, a luminance-defined edge should be defined not only by its direction and end point, but also by its curvature.

Another issue is that the current definition of relatability is all or none: A pair of edges either does, or does not, satisfy the two conditions that define relatability (Definition 1). Psychophysical data, however, show gradedness in subjects' responses (Kellman \& Shipley, 1991; Shipley \& Kellman, 1992a). For example, the perceived

(a)

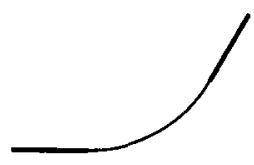

(b)

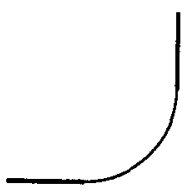

(c)

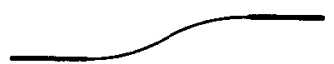

(d)

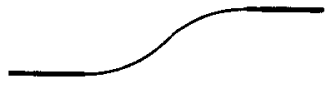

(e)

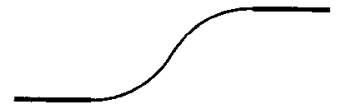

(f)

Figure 7. A graded measure of relatability based on $\int_{0}^{L}(d \kappa / d s)^{2} d s$ and $\int_{0}^{L}\left|\kappa_{\alpha}(s)\right| d s$ can track the gradual decrease in subjects' responses with increase in angle between two relatable edges $(a-c)$, and with increase in offset between two parallel edges $(d-f)$. strength of boundary interpolation decreases gradually with increasing angle (see Figures $7 \mathrm{a}-7 \mathrm{c}$ ) between a pair of relatable edges (Kellman \& Shipley, 1991). Moreover, for edge pairs that are parallel (see Figures 7d-7f), perceived interpolation strength decreases gradually with increasing degree of offset between the edges (Shipley \& Kellman, 1992a), even though only the colinear pair is relatable. Also, for textures composed of Gabor patches, the detectability of virtual lines connecting the patches decreases with increasing angle between the patches (Field, Hayes, \& Hess, 1993); similar effects have been found with textures composed of dots (Pizlo, Salach-Golyska, \& Rosenfeld, 1997; Smits \& Vos, 1987; Uttal, 1975). Hence, it would be desirable to have a measure of relatability that is graded. Takeichi et al.'s (1995) framework does allow for some gradedness: The more inflections an interpolated curve needs to have, while maintaining continuity of direction, the weaker the interpolation is. However, for the two cases mentioned above (see Figure 7), even this measure does not explain the gradedness in perceived strength of interpolation: For the relatable edge pairs shown in Figures $7 a-7 c$, the number of inflection points needed on the interpolated contour is zero -irrespective of the angle between the edges. Similarly, for the parallel edge pairs shown in Figures $7 d-7 f$, each interpolated contour needs exactly one point of inflection-irrespective of the degree of (nonzero) offset between the edges.

In the computer vision literature, a number of measures of smoothness for curves (and surfaces) have been proposed, and some of these have also been applied to perceptual psychology (e.g., Blake \& Zisserman, 1987; Grimson, 1981; Sha'ashua \& Ullman, 1988; Ullman, 1976; Weiss, 1990). A commonly used measure is variation of curvature. For a curve defined on $[0, L]$, the variation of curvature is given by

$$
\int_{0}^{L}\left(\frac{d \kappa_{\alpha}}{d s}\right)^{2} d s
$$

The lower this variation, the smoother the curve. Hence, interpolated curves are typically chosen in order to minimize this (or some such similar) measure.

One way to develop a graded notion of relatability is as follows: Given two edges, first choose a maximally smooth curve that interpolates between them-say, by minimizing variation of curvature. (This will also minimize the number of inflection points on the curve.) Then, for this curve, use the values

$$
\int_{0}^{L}\left|\kappa_{\alpha}(s)\right| d s
$$

(the measure used in Condition 3 of the proposition) and

$$
\int_{0}^{L}\left(\frac{d \kappa_{\alpha}}{d s}\right)^{2} d s
$$

(variation of curvature) to obtain a graded measure of relatability. Note that these two measures can vary inde- 
pendently of each other, and that both may be determinants of the degree of relatability. For example, the curves in Figures $7 \mathrm{a}-7 \mathrm{c}$ all have

$$
\int_{0}^{L}\left(\frac{d \kappa_{\alpha}}{d s}\right)^{2} d s=0
$$

but they differ in

$$
\int_{0}^{L}\left|\kappa_{\alpha}(s)\right| d s
$$

(the values being $0, \pi / 3$, and $\pi / 2$, respectively). Conversely, the curves in Figures $7 \mathrm{c}$ and $7 \mathrm{e}$ both have

$$
\int_{0}^{L}\left|\kappa_{\alpha}(s)\right| d s=\frac{\pi}{2}
$$

- that is, they both turn through a total angle of $\pi / 2$, but they differ in

$$
\int_{0}^{L}\left(\frac{d \kappa_{\alpha}}{d s}\right)^{2} d s
$$

(which gives value 0 for Figure $7 \mathrm{c}$, but a positive value for Figure 7e).

A graded measure of relatability based on both

$$
\int_{0}^{L}\left|\kappa_{\alpha}(s)\right| d s
$$

and

$$
\int_{0}^{L}\left(\frac{d \kappa_{\alpha}}{d s}\right)^{2} d s
$$

is consistent with the currently available data on boundary interpolation that shows gradedness in subjects' responses. It can explain the decrease in perceived strength of boundary interpolation with both (1) increase in angle between the two edges (Figures 7a-7c), and (2) increase in offset between two parallel edges (Figures $7 d-7 f$ ). It can also explain why having more inflection points on an interpolating curve decreases its perceived strength: Adding inflection points on a curve increases both

$$
\int_{0}^{L}\left|\kappa_{\alpha}(s)\right| d s
$$

and

$$
\int_{0}^{L}\left(\frac{d \kappa_{\alpha}}{d s}\right)^{2} d s
$$

Furthermore, in the case of parallel edges,

$$
\int_{0}^{L}\left|\kappa_{\alpha}(s)\right| d s
$$

provides a scale-invariant measure of the degree of offset between the two edges. Such a measure is desirable, because, within a reasonable range of scalings, one does not expect the perceived strength of interpolation (and hence, the degree of relatability) to change with scaling (e.g., if the entire figure is expanded to twice-or half-its original size). The precise way in which these two measures combine in order to give an overall measure of relatability remains, however, a matter of empirical investigation.

\section{CONCLUSION}

Relatability and minimizing inflections are important factors in determining whether or not human vision will interpolate a contour between two luminance-defined edges. They can also serve to formalize the notion of "good continuation" (see, e.g., Kellman \& Shipley, 1991). We have stated and proved a proposition that shows that, under appropriate conditions, relatability is mathematically equivalent to the existence of a smooth curve with no inflection points that interpolates between two edges. The conditions in the proposition thus provide an alternate definition of relatability, in terms of properties that an interpolating curve must satisfy. This characterization is useful because it allows for an easy way to extend the definition of relatability (1) to include the role of genericity, and (2) to extend the current all-or-none character of relatability to a graded measure that can track the gradedness in psychophysical data.

\section{REFERENCES}

Albert, M. K., \& Hoffman, D. D. (1995). Genericity in spatial vision. In R. D. Luce, M. D'Zmura, D. D. Hoffman, G. J. Iverson, \& A. K. Romney (Eds.), Geometric representations of perceptual phenomena (pp. 95-112). Mahwah, NJ: Erlbaum.

BIEDERMAN, I. (1987). Recognition-by-components: A theory of human image understanding. Psychological Review, 94, $115-147$

BINFORD, T. O. (1981). Inferring surfaces from images. Artificial Intelligence, 17, 205-244.

BlaKe, A., \& Zisserman, A. (1987). Visual reconstruction. Cambridge, MA: MIT Press.

Brainard, D. H., \& Freeman, W. T. (1997). Bayesian color constancy. Journal of the Optical Society of America A, 14, 1393-1411.

Field, D. J., HAYES, A., \& Hess, R. F. (1993). Contour integration by the human visual system: Evidence for a local "association field." $V i$ sion Research, 33, 173-193.

Freeman, W. T. (1994). The generic viewpoint assumption in a framework for visual perception. Nature, 368, 542-545.

Grimson, W. E. L. (1981). From images to surfaces: A computational study of the human early visual system. Cambridge, MA: MIT Press.

Hoffman, D. D. (1998). Visual intelligence: How we create what we see. New York: Norton.

Hoffman, D. D., \& Richards, W. A. (1984). Parts of recognition. Cognition, 18, 65-96.

HoffMan, D. D., \& Singh, M. (1997). Salience of visual parts. Cognition, 63, 29-78.

Kanizsa, G. (1979). Organization in vision. New York: Praeger.

Kellman, P. J., \& ShIPLEy, T. F. (1991). A theory of visual interpolation in object perception. Cognitive Psychology, 23, 141-221.

Michotte, A., Thines, G., \& CrabBe, G. (1991). Amodal completion of perceptual structures. In G. Thines, A. Costall, \& G. Butterworth (Eds.), Michotte's experimental phenomenology of perception (pp. 140-167). Hillsdale, NJ: Erlbaum. (Original work published 1964)

Millman, R. S., \& Parker, G. D. (1977). Elements of differential geometry. Englewood Cliffs, NJ: Prentice-Hall.

Pizlo, Z., Salach-Golyska, M., \& Rosenfeld, A. (1997). Curve detection in a noisy image. Vision Research, 37, 1217-1241.

Sha'ASHUA, A., \& Ullman, S. (1988). Structural saliency: The detection of globally salient structures using a locally connected network. 
In Proceedings of the Second International Conference on Computer Vision (pp. 321-327). Washington, DC: IEEE Computer Society Press.

Shipley, T. F., \& Kellman, P. J. (1992a). Perception of partly occluded objects and illusory figures: Evidence for an identity hypothesis. Journal of Experimental Psychology: Human Perception \& Performance, 18, 106-120.

Shipley, T. F., \& Kellman, P. J. (1992b). Strength of visual interpolation depends on the ratio of physically specified to total edge length. Perception \& Psychophysics, 52, 97-106.

Singh, M., \& HoffMan, D. D. (1997). Constructing and representing visual objects. Trends in Cognitive Sciences, 1(3), 98-102.

Singh, M., \& HoffMan, D. D. (1998). Part boundaries alter the perception of transparency. Psychological Science, 9, 370-378.

Singh, M.,, Hoffman, D. D., \& AlBerT, M. (in press). Contour completion and relative depth: Petter's rule and support ratio. Psychological Science.

Singh, M., Seyranian, G., \& Hoffman, D. D. (1999). Parsing silhouettes: The short-cut rule. Perception \& Psychophysics, 61, 636-660.

Smits, J. T. S., \& Vos, P. G. (1987). The perception of continuous curves in dot stimuli. Perception, 16, 121-131.

Takeichi, H., Nakazawa, H., Murakami, I., \& Shimojo, S. (1995). The theory of the curvature-constraint line for amodal completion. Perception, 24, 373-389.

Ullman, S. (1976). Filling-in the gaps: The shape of subjective contours and a model for their generation. Biological Cybernetics, 25, 1-6.

UTTAL, W. (1975). An autocorrelation theory of form detection. Hillsdale, NJ: Erlbaum.

WeISs, I. (1990). Shape reconstruction on a varying mesh. IEEE Transactions on Pattern Analysis \& Machine Intelligence, 12, 345-362.

\section{NOTES}

1. While motivating their definition of relatability, Kellman and Shipley (1991) point to the need for a monotonicity constraint: "Intuitively, it requires that the connection must progress continuously from one edge to the other. The connection cannot extend outward and then return, or double back on itself, etc." (p. 174). Their example of violation of the monotonicity constraint shows a curve with constant sign of curvature that turns through a full circle and a half, and which crosses itself (Figure 22, p. 174). They go on to say that the monotonicity constraint is incorporated formally in the two conditions that define relatability (Definition 1). Therefore, we shall simply use the two conditions in Definition 1 as defining relatability.

Kellman and Shipley (1991) also offer a different formulation of relatability (given that the edges are not parallel)-namely, $0 \leq R \cos \phi \leq r$ (see Figure 2), where $R$ and $r$ are chosen so that $R \geq r(\mathrm{p} .176)$. They assert that this is equivalent to Definition 1. Again, therefore, we shall simply use Definition 1 for relatability.

2 . The idea of minimizing the number of inflection points on an interpolating contour is perfectly consistent with standard approaches to curve interpolation in computer vision that minimize variation in curvature. For example, curves that minimize

$$
\int\left(\frac{d \kappa_{\alpha}}{d s}\right)^{2} d s
$$

also minimize number of inflection points.

3. However, the length of the edges may affect a different factor, called support ratio (Shipley \& Kellman, 1992b; see also Singh, Hoffman, \& Albert, in press).

4. In Appendix B of Kellman and Shipley (1991), Skeath uses this construction to show that "when two edges meet the relatability criterion, a first-order continuous curve can be fit between them, tangent to the end point at both edges" (p. 176). This is undoubtedly true (and the construction gives a concrete example). However, it is not a criterion that can serve to characterize relatability, since a first-order continuous curve can also be fit between two edges that are not relatable. The purpose of our proposition is to give just such a characterization-one that is both necessary and sufficient.

5. For any $C^{1}$ plane curve $\alpha$ defined on some interval $[0, L]$, it is always possible to define $\theta(s)$ such that $\theta$ is continuous on $[0, L]$ (see, e.g., Millman \& Parker, 1977 , p. 55). In general, this may involve adding integral multiples of $2 \pi$ in places, in order to ensure continuity; but this is not the case here.

6. These two cases are independent. Guaranteeing that the end points of the interpolated contour are not points of $C^{2}$ discontinuity does not guarantee that they will not be points of inflection-and vice versa.

(Manuscript received February 17, 1998; revision accepted for publication July 30,1998 .) 